\title{
A HIGH-POWER SOURCE OF OPTICAL RADIATION WITH MICROWAVE EXCITATION
}

\author{
G. Churyumov ${ }^{1,2}$, A. Denisov ${ }^{1}$, T. Frolova ${ }^{2}$, N. Wang ${ }^{1}$, J. Qiu $^{1}$ \\ ${ }^{1}$ 1Harbin Institute of Technology, 92, West Dazhi Street, Nan Gang District, Harbin, China \\ 2 Kharkiv National University of Radio Electronics, 14, Nauky Ave., 61166, Kharkiv, \\ Ukraine \\ g.churyumov@ukr.net
}

Keywords: microwave heating, magnetron, electrodeless sulfur lamp, plasma, microwave excitation

\section{Introduction}

For more than 50 years, interest to the microwave heating technology has not weakened. In addition to the traditional areas of its application, which described in detail in [1], recently there has been an expansion of technological possibilities for the use of microwave energy associated with the impact of electromagnetic waves of the microwave range on various materials (sintering of metal and ceramic powders) and media, including plasma [2]. One such new direction is the creation of high-power and environmentally friendly sources of optical radiation on the basis of the electrodeless sulfur lamp with microwave excitation [2, $3]$.

As it is known, Michael Ury and his associates at Fusion Systems invented this radically new lamp in 1990, but the lamp was not ideal because of the complexity of its design [4]. Therefore, it was not put into production. However, every year the scientific interest was growing. An analysis of scientific publications shows that every 5 years a new country is joined to this issue. Now more than in 10 countries of all would including USA, Great Britain, South Korea, Netherlands, Germany, Russia, and so on where there are research teams that are carried out an investigation concerning the electrodeless lamps with microwave excitation.

The plasma lighting plant with a sulfur lamp is a high-power light source providing light fluxes of $\sim 140 \mathrm{klm}$, and color temperature of about $6400 \mathrm{~K}$. The main advantage of such lamps is its quasi-solar emission spectrum as well as the ability controlling by radiation power in process of its operation. As a result, it allows imitating the modes of sunrise and sunset and may be of interest in future for creating the solar simulator [5]. The main spectral and color characteristics of the electrodeless sulfur lamps with microwave excitation are presented in Figs. 1 and 2. For comparison in the same place the standard solar spectrum AM $1.5 \mathrm{G}$ is shown.

The purpose of this paper is to the further development of the theory and practice of microwave excitation by the electrodeless sulfur lamps, improving the energy efficiency during energy conversion into the optical radiation and widening the application of new light sources in real practice.

\section{Physical processes in the two-component microwave plasma}

The principle of ionization of gases under the influence of microwave energy is the base for the plasma lamp. Microwaves generated by the magnetron excites sulfur vapor in argon within the bulb. Highly ionized gas becomes a plasma state, which begins to emit light constantly when a certain operating temperature is reached. 


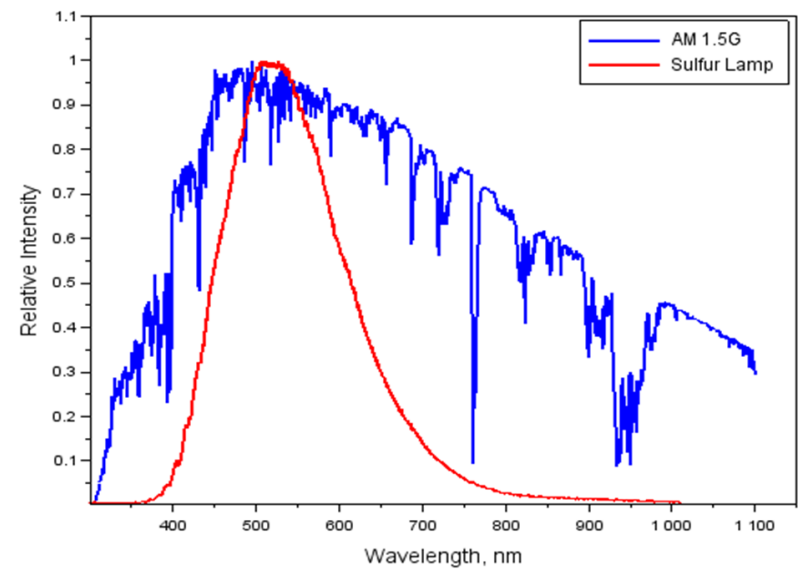

Fig. 1. Spectral characteristics of the electrodeless sulfur lamp with microwave excitation and spectral profile of the standard solar radiation [6]

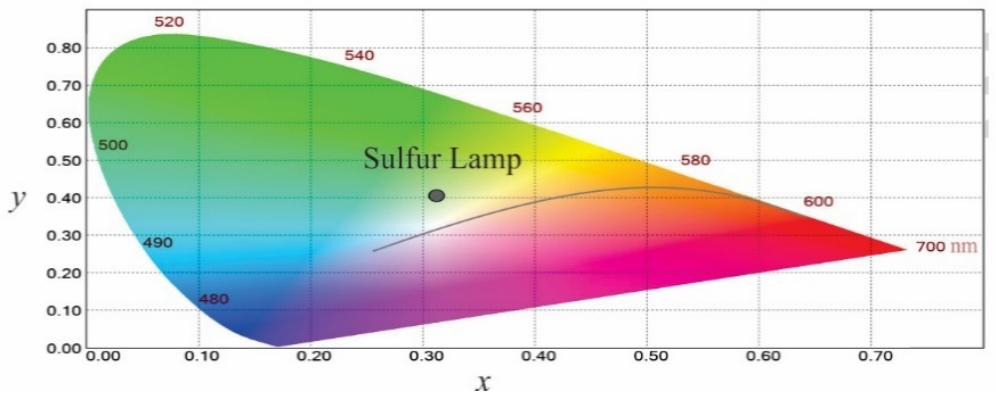

Fig. 2. Color characteristics of the electrodeless sulfur lamp with microwave excitation

For understanding the mechanism of converting microwave energy into optical radiation and for further improvement of the characteristics optical radiation sources it is necessary to study the physical processes occurring in the spherical quartz bulb filled with an inert gas (e.g., Ne or Ag) and having a small number of main component (sulfur in solid state). Impacting the microwave radiation causes a glowing discharge in the buffer gas having a low breakdown threshold on low initial pressure. At the same time with an increase in the quartz bulb filling temperature, the state of sulfur changes from solid to gaseous and at temperatures greater than $600 \mathrm{C}$, most of the sulfur has dissociated to $\mathrm{S}_{2}$ [7]. In the resulting high-pressure gas mixture, the microwave field converts the atoms (as well as dimers and other particles) of sulfur to excited states. When this occurs, the processes of direct atomic and molecular absorption, and the collisional mechanism. Finally, reradiation from excited states forms the observed optical emission spectrum.

Characteristic lines in the spectrum of the sulfur atom are in the open portion of the optical spectrum of a molecule 867, 921 and $1045 \mathrm{~nm}$ [8]. The ionization potential of each radiating state is less than $3 \mathrm{eV}$. To get some idea of the energetic interactions between sulfur dimer and atom, consider a simplified diagram of the energy levels of the two systems which shown in Fig. 3. As illustrated by Fig. 3, we use the dissociation energy of the ground state of the dimer, and $4.4 \mathrm{eV}$ - energy offset between the systems. 


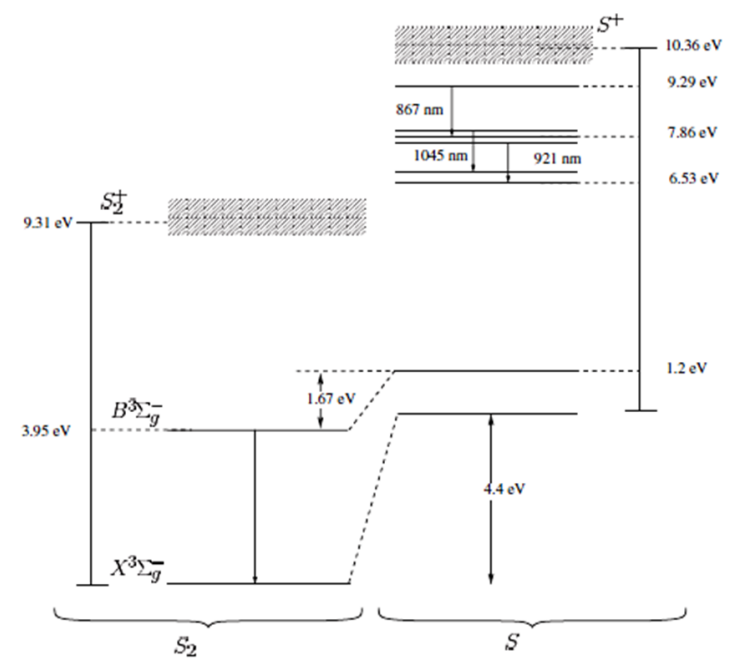

Fig. 3. The diagram of the energy levels of sulfur [8]

The ionization energy of the molecule is more attractive since the ionization potential of the molecular state is less than half of the ground state of the atom. Thus, if the degree of dissociation is relatively low, $\mathrm{S}_{2}{ }^{+}$ions will be the dominant species. As the level of the emitting atoms is about $11 \mathrm{eV}$ and higher molecular state, and their density is exponentially sensitive to temperature, they are ideal for study.

Studies show that the emission spectrum of the sulfur lamp in the visible region is formed mainly by radiative transitions between the excited level $B^{3} \sum \bar{u}$ and the ground state $X^{3} \sum \bar{g}$ of diatomic sulfur molecules $\mathrm{S}_{2}$.

\section{Mathematical description of the states of plasma}

Due to the diversity of processes occurring in spatially inhomogeneous plasma, an analytical description of the actual plasma is generally very difficult. Therefore, it is usually considered a simplified model of plasma, and then specifying how real plasma is close to the accepted model.

Condition of real plasma in an arbitrary pressure is determined by:

a) the concentrations of particles of all kinds (the number of particles in unit volume);

b) their velocities distribution function;

c) the population of excited levels (the number of particles per unit volume in the excited state);

d) the spatial distributions of these quantities.

Getting information on all the above characteristics is extremely difficult, as the theoretical studies of the plasma state require setting up and solving the system of equations connecting these quantities with the external conditions.

For description inside an electrodeless sulfur lamp of gas-discharge plasma, formed as a result impacting the electromagnetic field, it is advisable to use the local thermodynamic equilibrium (LTE) model. This model allows us to qualitatively and quantitatively describe the continuous spectrum of the lamp, as well as the distribution of basic physical quantities of sulfur plasma. 
According to the model of the LTE temperature in the different elements of the volume of the medium is different, but the plasma can be described (locally) by dint of the Boltzmann and Maxwell distributions and Saha equation in each volume element where the local temperature is the same for all types of particles.

The LTE model for most stationary cases of generating plasmas received in the laboratory conditions is typical. Under the conditions of the LTE model of plasma, the detailed equilibrium for optical transitions it is broken, so it is advisable to consider radiation and absorption separately.

\section{Description of the software product}

The optical radiation source may present itself a spherical or cylindrical flask of transparent anhydrous quartz glass filled with metered amounts of sulfur (and possibly to include contaminants, such as $\mathrm{CaBr} 2$ ) or another substance (e.g. indium iodide $\mathrm{InI}$ ) as well as buffer gas (argon, neon, krypton). Theoretical studies of output spectral characteristics of the optical radiation of such source and their dependence on the microwave power to the pump wavelength from $\lambda$ to $\lambda+d \lambda$, the temperature distribution inside the bulb, the electrical conductivity of the plasma, and so on can be carried out by changing the composition of the bulb filling.

The buffer gas (argon) is used to obtain the initial ionization and glow discharge (gas pressure is set at the initial stage). We get the dependence of the dynamics of change of pressure in the bulb temperature.

The interface developed software named HID Lamps v.2.0 is shown in Fig. 4. All output parameters and characteristics of the charts have the opportunity to be saved in separate files for further analysis and comparison of the results with experimental data.

The simulation was performed for the electrodeless discharge lamps filled with sulfur $\mathrm{S}$ and indium iodide InI as active components with the buffer gas argon or neon. We studied the effect of the amount of filling in the luminous efficacy and color rendering index behavior. These results were tested and experimental studies. However, it was noticed that there are threshold values for the buffer gas below and above which can not be taken since it is impossible to receive the gas discharge. For easier ignition, it is necessary to choose a pressure of the buffer gas close to the lower boundary.

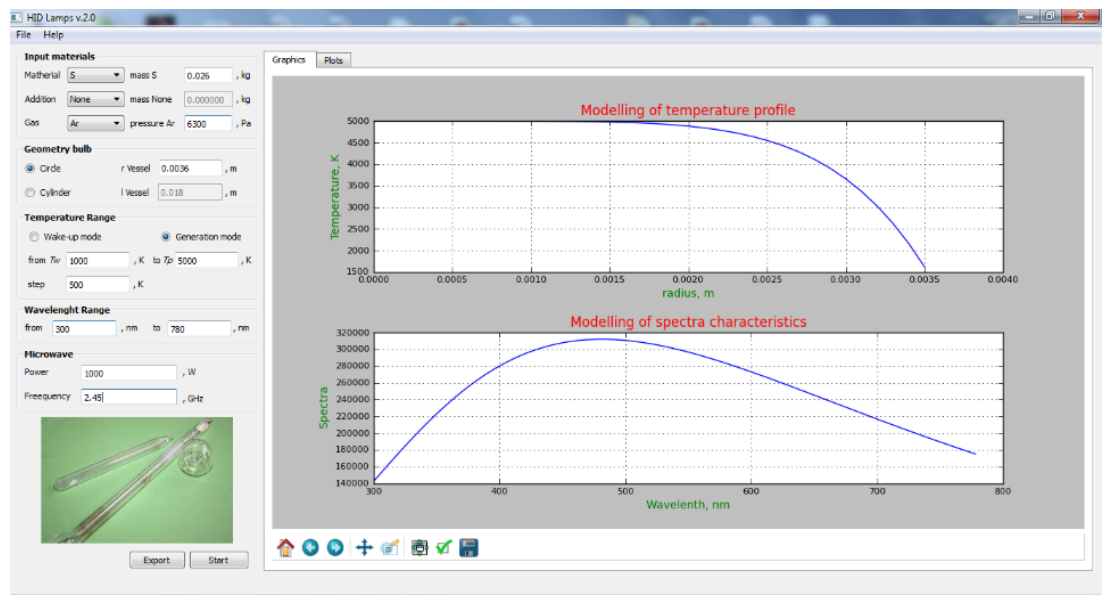

Fig. 4. The interface of the software HID Lamps v.2.0 
There were also carried out the theoretical studies of the emission spectrum depending on the type of microwave pumping source (microwave devices), the pressure in the flask and the concentration of the substance. As a result, the following assumption can be made that using the LTE model to describe the gas-discharge plasma, the pressure change will primarily affect the spectral characteristics of the system. Therefore, the work was to study the behavior of the spectrum lamp for different values of the buffer gas pressure of argon.

\section{Vistas of application}

The electrodeless sulfur lamps with microwave excitation can be used together with other electronic devices for creating the power energy-efficient lighting systems. It is proposed to use a lighting facility based on an electrodeless sulfur lamp with microwave excitation combined with solar panels that are located indoors (for example, greenhouses) [9]. This gives several advantages: first, it facilitates the maintenance of solar panels (protection from various natural phenomena); secondly, with the reduction in the number of sunny days, increases the need to use artificial light (for example, for supplementary lighting plants in greenhouses), however, if the lighting devices have a spectrum close to the Sun, then solar panels can more efficiently produce electricity.

It is shown that additional use of the solar panels for the reverse conversion of the optical radiation into DC energy with follow-up its using in the circuits of secondary power supply allows improving the energy efficiency of the light source.

Table 1 compares the photovoltaic characteristics of the solar panels under solar irradiation $(1000 \mathrm{~W} / \mathrm{m} 2)$ and the sulfur lamp (170 W/ m2).

Table 1. Comparison photovoltaic parameters.

\begin{tabular}{ccccccc}
\hline \hline \multirow{2}{*}{ Light source } & \multicolumn{7}{c}{ Parameters } \\
\cline { 2 - 7 } & $\boldsymbol{I}_{\boldsymbol{s c}, \boldsymbol{A}}$ & $\boldsymbol{V}_{\boldsymbol{o c}}, \boldsymbol{W}$ & $\begin{array}{c}\boldsymbol{J}_{\boldsymbol{s c}}, \\
\boldsymbol{A} / \boldsymbol{m}^{2}\end{array}$ & $\eta, \boldsymbol{\%}$ & $\boldsymbol{F F}$ & $\boldsymbol{P}_{\text {out }}, \boldsymbol{W}$ \\
\hline Outdoor (Sun) & 8,94 & 38,5 & 5,5 & 16,6 & 0,78 & 165,2 \\
Sulfur Lamp & 0,97 & 36,4 & 0,6 & 8,15 & 0,79 & 17,3 \\
\hline \hline
\end{tabular}

The fill factor finds as

$$
F F=\frac{V_{m} \cdot I_{m}}{V_{O C} \cdot I_{s c}}=\frac{P_{\max }}{V_{O C} \cdot I_{s c}},
$$

where $V_{m}$ - the voltage and current at peak power, respectively; $P_{\max }$ - the maximum output power of solar cell; $V_{O C}-$ the open circuit voltage; $I_{S C}-$ the short circuit current. The solar efficiency of the solar cell can be written as

$$
\eta=\frac{V_{m} \cdot I_{m}}{S \cdot E} \times 100 \%
$$

where $S-$ the area of solar cell on which radiation falls $\left[\mathrm{m}^{2}\right] ; E-$ the irradiance $\left[\mathrm{W} / \mathrm{m}^{2}\right]$. 
The electrical power output of the solar cell is given by

$$
P_{\text {out }}=F F \cdot J_{S C} \cdot V_{o C}
$$

Fig. 5 shows the thermal images taken from the back solar panel under the real sun and the electrodeless sulfur lamp with microwave excitation.
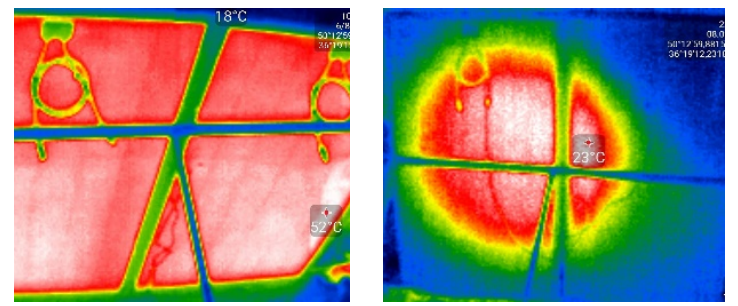

Fig. 5. Thermal imaging of the solar panel with the back side

When using a tungsten halogen lamp together with a sulfur lamp, the spectral response of solar cells in the near-infrared region can be improved. In Fig. 6 shows the spectra of sulfur and halogen lamps separately and their combined radiation, as well as the external quantum efficiency for silicon.

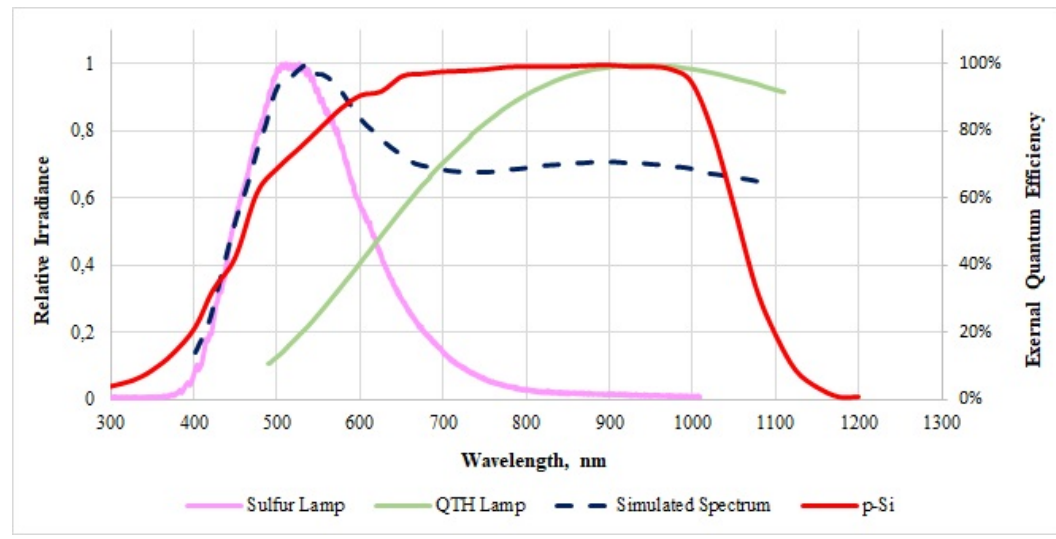

Fig. 6. Spectral profiles of lamps and spectral response curve for silicon

The Fig. 7 and 8 show a general block diagram of a lighting device based on the connection of an electrodeless sulfur lamp with microwave excitation to solar batteries and a diagram of connecting solar panels with additional devices: the solar panels, a charge controller, the batteries, an inverter, an automatic transfer switch, an electric network, a light systems and other electronic devices. 


\section{Conclusions}

Thus, an application of the electrodeless sulfur lamps with microwave excitation allows creating new electronic plants and systems for the effective decision of different technology problems. It is shown, that the output optical radiation of an electrodeless sulfur lamp has a

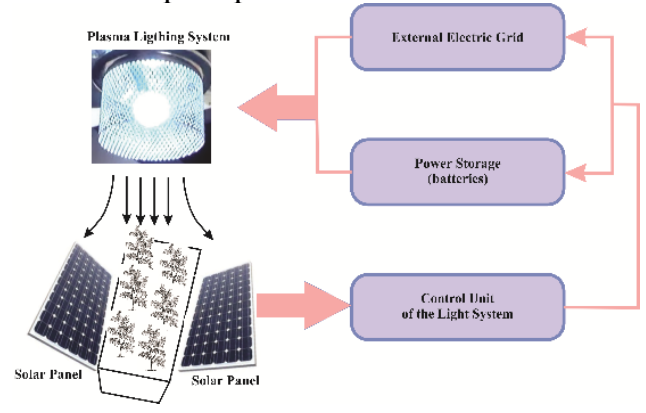

Fig. 7. General block diagram of a lighting device on the basis of linking an electrodeless sulfur lamp with microwave excitation and solar panels

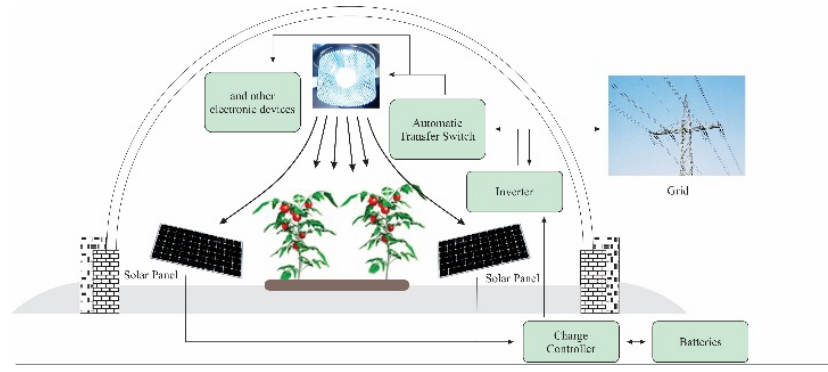

Fig. 8. Scheme of connection of the solar panels

continuous quasi-solar spectrum close to the spectrum of the Sun in the wavelength interval, where the spectral sensitivity of the solar cells is the most effective. The possibility of using plasma lighting systems based on an electrodeless solar lamp with microwave excitation in combination with solar batteries has an additional regeneration of electricity by converting its optical radiation into electric current using solar panels. Regenerated energy can be used in the future for the operation of both the lighting device itself and other electrical equipment or for its return to the external electrical grid. This will save energy and reduce the cost of production in general (for example, when growing agricultural products).

\section{References}

1. Microwave Power Engineering. Edited by E.C. Okress. V. 1, 2. Academic Press, New York \& London. 1968.

2. A. N. Didenko, SVCh-energetika. Teoriya i praktika. - Moscow: Nauka. 2003. $445 \mathrm{~s}$.

3. G. Churyumov, T. Frolova, "Microwave Energy and Light Energy Transformation: Methods, Schemes and Designs. Microwave Energy and Light Energy Transformation: Methods, Schemes and Designs" // In the book "Emerging Microwave Technologies in Industrial, Agricultural, Medical and Food Processing.” Edited by Kok Yeow You, IntechOpen, 2018. pp. 75-91. 
4. Dolan I.T., Ury M.G., Wood C.H. A Novel High Efficacy Microwave Powered Light Source. Presented as a Land-mark.- In: Sixth International Symposium on the Science and Technology of Light Sources, 1992, Technical University of Budapest.

5. Frolova T. Analysis of a Solar Simulator Based on the Electrodeless Sulfur Lamp for Photovoltaic Devices // T. Frolova, A. Frolov / Telecommunications and Radio Engineering, № 77 (6), - 2018. - P. 525-539. DOI: 10.1615/TelecomRadEng.v77.i6.50

6. ASTM G173-03(2012), Standard Tables for Reference Solar Spectral Irradiances: Direct Normal and Hemispherical on $37^{\circ}$ Tilted Surface, ASTM International, West Conshohocken, PA, 2012, DOI: 10.1520/G0173-03R12

7. D.A. Peterson, L.A. Schlie. Stable pure sulfur discharges and associated spectra // The Journal of Chemical Physics, Vol. 73, \# 4. 1980. P. 1551-1566.

8. C. W. Johnston, J. Jonkers, and J. J. A. M. van der Mullen, "Operational trends in the temperature of a high-pressure microwave powered sulfur lamp," J. Phys. D: Appl. Phys., 2002. pp. 2578-2585.

9. Frolova T.I., Churyumov G.I. Lighting Devices based on the Electrodeless Sulfur Lamp with Microwave Excitation / Patent of Ukraine \# 123127; Publication 12 February 2018 (2018) (in Ukrainian) 